\title{
Electrical stimulation therapy of the lower esophageal sphincter is successful in treating GERD: final results of open-label prospective trial
}

\author{
Leonardo Rodríguez $\cdot$ Patricia Rodriguez $\cdot$ Beatriz Gómez $\cdot$ Juan C. Ayala $\cdot$ \\ Jorge Saba $\cdot$ Alberto Perez-Castilla $\cdot$ Manoel Galvao Neto $\cdot$ Michael D. Crowell
}

Received: 27 June 2012/ Accepted: 21 August 2012/Published online: 17 October 2012

(C) Springer Science+Business Media, LLC 2012

\begin{abstract}
Background Electrical stimulation of the lower esophageal sphincter (LES) improves LES pressure without interfering with LES relaxation. The aim of this open-label pilot trial was to evaluate the safety and efficacy of longterm LES stimulation using a permanently implanted LES stimulator in patients with gastroesophageal reflux disease (GERD).

Methods GERD patients who were at least partially responsive to proton pump inhibitors (PPI) with abnormal esophageal $\mathrm{pH}$, hiatal hernia $\leq 3 \mathrm{~cm}$, and esophagitis $\leq \mathrm{LA}$ grade $\mathrm{C}$ were included. Bipolar stitch electrodes were placed in the LES and an IPG was placed in a subcutaneous pocket. Electrical stimulation was delivered at $20 \mathrm{~Hz}, 215$ $\mu \mathrm{s}, 3-8 \mathrm{~mA}$ in $30 \mathrm{~min}$ sessions. The number and timing of sessions was tailored to each patient's GERD profile. Patients were evaluated using GERD-HRQL, daily symptom and medication diaries, SF-12, esophageal $\mathrm{pH}$, and high-resolution manometry.
\end{abstract}

Presented at the SAGES 2012 Annual Meeting, San Diego, CA, March 7-10, 2012.

L. Rodríguez $(\bowtie) \cdot$ P. Rodriguez · B. Gómez

Department of Surgery, Centro Clínico de Obesidad (CCO),

Obesidad Y Diabetes, Estoril $N^{\circ} 120$ Of. 814, Las Condes,

Santiago, Chile

e-mail: doctor@leonardorodriguez.cl

J. C. Ayala $\cdot$ J. Saba $\cdot$ A. Perez-Castilla

Department of Gastroenterology, Clinica Indisa, Santiago, Chile

M. Galvao Neto

Department of Surgery, Gastro Obeso Center, São Paulo, Brazil

M. D. Crowell

Mayo Clinic in Arizona, Scottsdale, AZ, USA
Results 24 patients (mean age $=53$ years, $\mathrm{SD}=12$ years; 14 men) were implanted; 23 completed their 6-month evaluation. Median GERD-HRQL scores at 6 months was $2.0(\mathrm{IQR}=0-5.5)$ and was significantly better than both baseline on-PPI [9.0 (range $=6.0-10.0$ ); $p<0.001]$ and off-PPI [23 (21-25); $p<0.001]$ GERDHRQL. Median\% 24-h esophageal $\mathrm{pH}<4.0$ at baseline was 10.1 and improved to 5.1 at 6 months $(p<0.001)$. At their 6-month follow-up, $91 \%(21 / 23)$ of the patients were off PPI and had significantly better median GERD-HRQL on LES stimulation compared to their on-PPI GERDHRQL at baseline (9.0 vs. $2.0 ; p<0.001)$. There were no unanticipated implantation- or stimulation-related adverse events or untoward sensation due to stimulation. There were no reports of treatment-related dysphagia, and manometric swallow was also unaffected.

Conclusions Electrical stimulation of the LES is safe and effective for treating GERD. There is a significant and sustained improvement in GERD symptoms, esophageal $\mathrm{pH}$, and reduction in PPI usage without any side effects with the therapy. Furthermore, the therapy can be optimized to address an individual patient's disease.

Keywords GERD · Electrical stimulation · Surgical treatment · Refractory GERD

Gastroesophageal reflux disease (GERD) is among the most common gastrointestinal disorders [1]. Heartburn afflicts nearly two-thirds of US adults at some point in their lives and accounts for 4-5 million physician office visits every year [2]. The total annual cost of care because of GERD in the US is estimated at $\$ 9.8$ billion, $\$ 5.8$ billion of which is spent on medications [1]. From a physiological perspective, GERD results from failure of the antireflux 
function of the lower esophageal sphincter (LES), allowing abnormal reflux of gastric contents into the esophagus [3]. Medical therapy with acid suppression fails to address this pathophysiology and bothersome symptoms related to weakly acidic or nonacidic reflux or nocturnal GERD persist in $38 \%$ of patients [4]. Laparoscopic fundoplication is currently the mainstay of surgical GERD therapy and is offered primarily to those in whom medical therapy fails. In high-volume centers, the efficacy of surgical fundoplication is comparable or better than that of chronic medical therapy [3]. However, due to concerns about long-term failure, morbidity, and long-term side effects associated with antireflux surgery, only a small fraction of GERD sufferers refractory to medical therapy are referred for surgery, leaving a significant number of these patients without an effective therapeutic option. This unmet medical need has led to multiple attempts at development of less invasive endoscopic and surgical devices for the treatment of GERD.

Electrical stimulation of the LES in both acute and chronic animal models has been shown to increase resting LES pressure [5-7]. Short-term electrical stimulation using temporary leads implanted in the LES in subjects with GERD has shown similar results without interfering with physiological LES relaxation, suggesting that this may be an effective method of restoring the antireflux function of diseased LES without interference with normal LES relaxation [8, 17].

The LES Stimulation System (EndoStim BV, the Hague, Netherlands) is an implantable electrical stimulator that delivers long-term electrical stimulation therapy to the
LES. The objectives of this open-label human trial were to assess the safety of chronic electrical stimulation of the LES and to evaluate the effect of such stimulation on GERD symptoms and medication use, esophageal acid exposure and esophageal motor function in patients with GERD.

\section{Materials and methods}

Study design and patient selection

This was a prospective, open-label, single-center, treatment-only trial. The study was approved by the Institutional Review Board of Clinica Indisa, Santiago, Chile. All subjects signed an informed consent form. The study's inclusion and exclusion criteria are listed in Table 1.

\section{LES stimulation system}

The LES Stimulation System comprises three components: a bipolar stimulation lead with two stitch electrodes, an implantable pulse generator (IPG), and an external programmer (Fig.2a). The stimulation lead is $45 \mathrm{~cm}$ long and has sterile, bipolar, stitch platinum-iridium electrodes at the end that are implanted in the LES. The IPG is of typical titanium case construction. The IPG contains a medicalgrade lithium battery, microelectronics, communication coils, and an accelerometer for sensing patient posture. It is hermetically sealed to prevent damage to the device from biological fluids. The IPG is connected to the stimulation

Table 1 Study inclusion and exclusion criteria

\begin{tabular}{|c|c|}
\hline Inclusion criteria & Exclusion criteria \\
\hline a. $21-65$ years of age & a. Non-GERD esophageal motility disorders or gastroparesis \\
\hline $\begin{array}{l}\text { b. Heartburn, regurgitation, or both for }>6 \text { months necessitating daily } \\
\text { use of PPI }\end{array}$ & $\begin{array}{l}\text { b. Subject has significant multisystem diseases (e.g., scleroderma, } \\
\text { dermatomyositis, CREST, Sjögren's, Sharp's syndrome, etc.) }\end{array}$ \\
\hline $\begin{array}{l}\text { c. Baseline GERD-HRQL heartburn score of } \geq 20 \text { off PPI with at least } \\
\text { 10-point improvement on PPI }\end{array}$ & c. Barrett $(>\mathrm{M} 2 ;>\mathrm{C} 1)$ or any dysplasia \\
\hline $\begin{array}{l}\text { d. American Society of Anesthesiologists (ASA) physical status } \\
\text { classification } \leq \text { II }\end{array}$ & d. Hiatus hernia $>3 \mathrm{~cm}$ \\
\hline $\begin{array}{l}\text { e. Distal esophageal } \mathrm{pH}<4 \text { on } 24 \text {-h pH-metry off antisecretory } \\
\text { therapy for }>5 \%\end{array}$ & e. Body mass index $>35 \mathrm{~kg} / \mathrm{m}^{2}$ \\
\hline $\begin{array}{l}\text { f. Resting LES EEP } \geq 5 \mathrm{mmHg} \text { and } \leq 15 \mathrm{mmHg} \text {; esophageal body } \\
\text { contraction amplitude }>30 \mathrm{mmHg} \text { for }>70 \% \text { of swallows and } \\
>50 \% \text { peristaltic contractions }\end{array}$ & $\begin{array}{l}\text { f. Type } 1 \text { diabetes mellitus or uncontrolled type } 2 \text { diabetes mellitus } \\
\text { (T2DM) defined as HbA1c }>9.5 \text { in the previous } 6 \text { months, or T2DM } \\
\text { for }>10 \text { years }\end{array}$ \\
\hline g. Esophagitis $\leq$ grade C (LA classification) & g. Suspected or confirmed esophageal or gastric malignancy or varices \\
\hline \multirow[t]{4}{*}{ h. Signed informed consent } & $\begin{array}{l}\text { h. Significant cardiac arrhythmia, ectopy, significant cardiovascular } \\
\text { disease }\end{array}$ \\
\hline & i. Implanted electromedical device (e.g., pacemaker) \\
\hline & j. Pregnancy \\
\hline & k. Esophageal or gastric surgery, including antireflux surgery \\
\hline
\end{tabular}




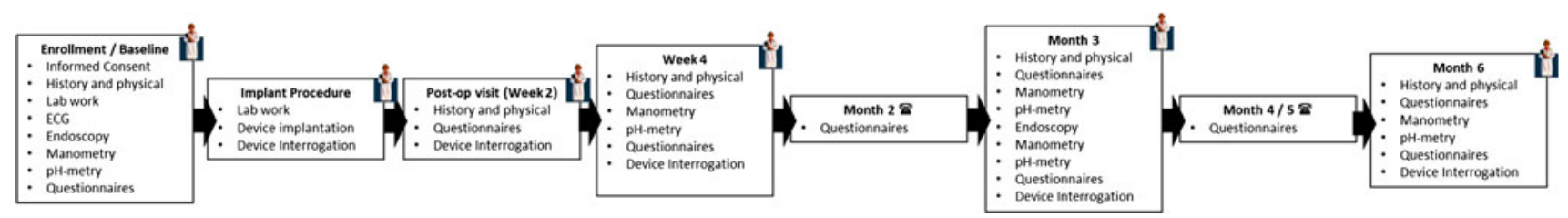

Fig. 1 Trial Design and Follow-up.

A

B
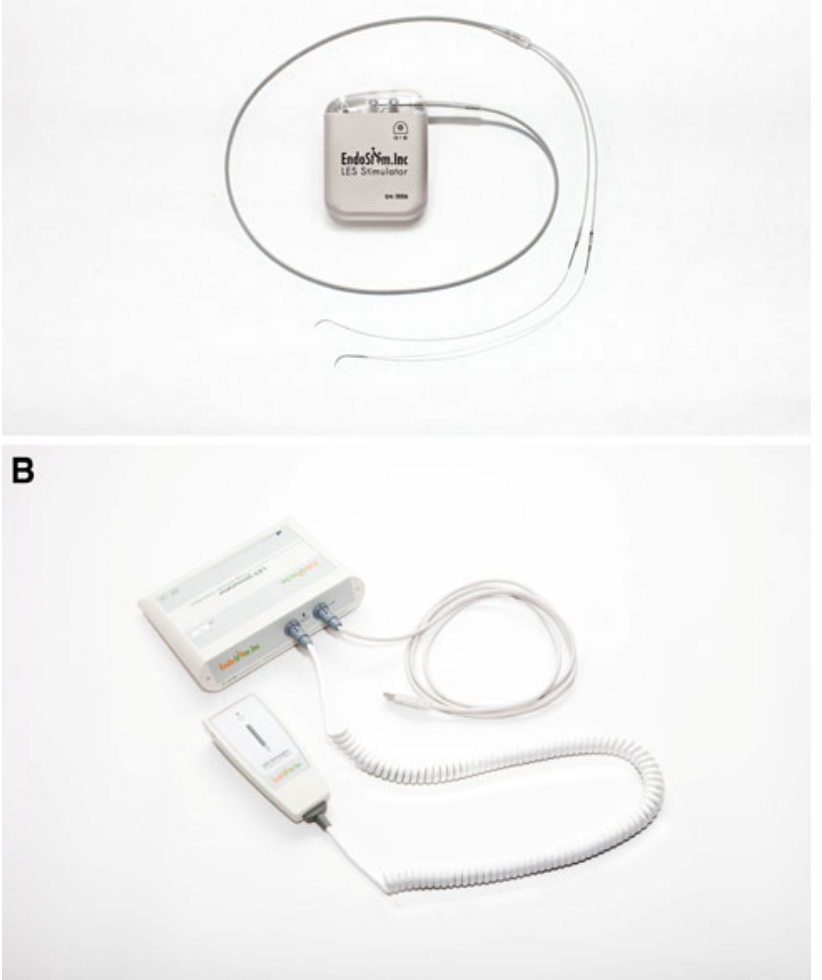

Fig. 2 a LES Stimulation System (EndoStim BV, the Netherlands) comprises an IPG $(65 \mathrm{~mm} \times 48 \mathrm{~mm} \times 12 \mathrm{~mm}$, weight $=49 \mathrm{~g})$ that is implanted in the subcutaneous pocket in the anterior abdomen and the bipolar electrodes are implanted in the LES and connected to the IPG. b The programmer is used to wirelessly program the IPG using a radiofrequency signal

leads and is permanently implanted in a subcutaneous pocket in the left upper quadrant of the abdomen. An external programmer, similar to other neurostimulator programmers, allows for wireless interrogation and programming of the IPG via laptop PC software (Fig. 2b).

LES stimulator implant procedure

The LES stimulator implant procedure is performed using standard laparoscopic techniques. The patient is positioned

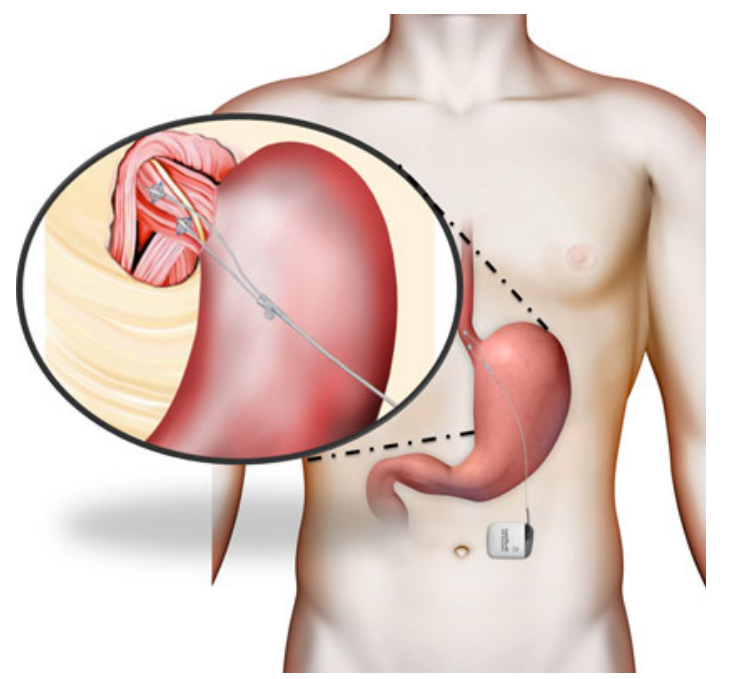

Fig. 3 LES electrode position and IPG implant location. Bipolar stitch electrodes are placed in the abdominal esophagus inline $1 \mathrm{~cm}$ apart. The lead is connected to the IPG that is implanted in the subcutaneous pocket in the anterior abdomen

in a mild reverse Trendelenburg position. Before scrubbing, the skin incision for the subcutaneous pulse generator is marked at $3-5 \mathrm{~cm}$ below the left costal line and parallel to it with lateral tendency. The abdomen is prepped and draped in the usual sterile manner. Entry to the abdominal cavity is gained using open, Veress needle, or optics-guided access at a supraumbilical position and pneumoperitoneum is induced.

Four trocars are placed under direct vision as follows: two working ports at the upper right quadrant, one in the subxiphoid region for a liver retractor, and one on the subcostal left anterior axillary line (preferably along the pulse generator's skin incision mark) for the assistant's instruments. One port needs to be a $10 \mathrm{~mm}$ port to allow for lead introduction into the abdominal cavity, and other ports could be 5 or $3 \mathrm{~mm}$ ports.

The anterior right aspect of the abdominal esophagus is exposed using a cautery hook or a harmonic scalpel and blunt dissection. Attention is paid to minimize thermal damage to any nerve branches in this area. The pars flaccida of the hepatogastric ligament as well as the 
paraesophageal fat pad are dissected to expose a rectangular longitudinal area of $3 \times 1 \mathrm{~cm}$. Attempts should be made to avoid dissection of the phrenoesophageal attachment and damage to the anterior vagal nerve. It is recommended that any hiatal hernia present be appropriately repaired using standard surgical techniques. If required, dissection of both crura for transhiatal mobilization of the distal esophagus so as to achieve $2-3 \mathrm{~cm}$ of tension-free abdominal esophagus for lead implantation followed by crural closure posteriorly with nonabsorbable sutures is recommended.

After endoscopic exploration of the esophagus, the Z-line is identified by transillumination. Placement of the electrodes is performed under endoscopic visualization to avoid perforation of the esophageal lumen.

The bifurcated bipolar lead is then completely introduced into the abdominal cavity. A left caudal retraction is applied to the gastric cardia by an assistant using an atraumatic grasper. The guiding needle of the first electrode is passed in the esophageal wall taking a $15 \mathrm{~mm}$ superficial longitudinal bite at the anterior right aspect of the esophagus above the Z-line, avoiding the anterior vagal branch as well as arterial and venous branches of the left gastric vessels. The electrode is placed in the muscularis propria of the LES. The second electrode is placed in similar fashion in an inline position and approximately $10 \mathrm{~mm}$ distal to the first electrode (Fig. 3).

Two titanium clips are placed on the nylon thread of each electrode as it comes out of the esophageal muscle. These clips serve as distal stoppers. The proximal part of each electrode is anchored to the underlying muscle using 2 or 3/0 multifilament, nonabsorbable thread which is applied at both sides of each silicone "butterfly." Repair of any hiatal hernia was not performed in this trial.

The abdomen is desufflated and the skin incision for the pulse generator is made. A subcutaneous pocket is created by blunt dissection. Pneumoperitoneum is reinduced and a small-caliber trocar is used to puncture the fascia only, through the skin incision, to pull the lead electrical connector toward the pulse generator's pocket. After thorough cleaning, the connector is attached to the pulse generator and a functionality test is performed by the technical support personnel. The pulse generator is placed into its pocket and excess lead is simultaneously pulled into the abdominal cavity and placed along the left abdominal wall away from the midline (Fig. 3).

The abdomen is desufflated and a final functionality test is performed. The subcutaneous tissue is carefully sutured above the pulse generator and the skin is sutured. The skin trocar wounds are treated and bandaged. The patient is discharged home after an overnight stay. It is recommended that the patient wear an elastic compression bandage over the pulse generator implantation site for
Table 2 Baseline patient characteristics and relevant medical/GERD history

\begin{tabular}{|c|c|c|}
\hline \multicolumn{2}{|l|}{ Characteristic } & \multirow{2}{*}{$\begin{array}{l}\text { Mean (SD) } \\
52(11.6)\end{array}$} \\
\hline Age (years) & 24 & \\
\hline Body mass index (BMI) & 24 & $27.8(3.2)$ \\
\hline \multicolumn{3}{|l|}{ Gender } \\
\hline \multicolumn{2}{|l|}{ Male } & 14 \\
\hline Female & & 10 \\
\hline \multicolumn{3}{|l|}{ BMI class } \\
\hline Normal $(<25)$ & & 3 \\
\hline Overweight $(\geq 25$ and $<$ & & 14 \\
\hline Obese $(\geq 30)$ & & 7 \\
\hline \multicolumn{2}{|l|}{ Patients using daily PPI } & $100 \%$ \\
\hline \multicolumn{2}{|c|}{ Duration of GERD symptoms [mean (SD)] } & $>11(8)$ years \\
\hline \multicolumn{2}{|l|}{ Median (quartile) } & $>10(7-14)$ years \\
\hline \multicolumn{2}{|c|}{ Duration of PPI use [mean (SD)] } & $>5.8(3.3)$ years \\
\hline \multicolumn{2}{|l|}{ Median (quartile) } & $>5.5(3-10)$ years \\
\hline GERD-HRQL total score & On PPI & Off PPI \\
\hline Mean (SD) & $10.4(6.8)$ & $23.7(3.5)$ \\
\hline Median (IQR) & $9(6-10)$ & $23.5(21-25.8)$ \\
\hline$\%$ not satisfied & 71 & 92 \\
\hline \multicolumn{3}{|l|}{ Heartburn frequency/week } \\
\hline Mean (SD) & $\mathrm{NC}$ & $93 \%(10)$ \\
\hline Median (IQR) & & $100 \%(86-100)$ \\
\hline \multicolumn{3}{|c|}{ Regurgitation frequency/week } \\
\hline Mean (SD) & \multirow[t]{2}{*}{$\mathrm{NC}$} & $67 \%(38)$ \\
\hline Median (IQR) & & $93 \%(30-98)$ \\
\hline \multicolumn{3}{|c|}{ Nocturnal heartburn frequency/week } \\
\hline Mean (SD) & \multirow[t]{2}{*}{$\mathrm{NC}$} & $59 \%(35)$ \\
\hline Median (IQR) & & $71 \%(29-89)$ \\
\hline \multicolumn{3}{|c|}{ Nocturnal regurgitation frequency/week } \\
\hline Mean (SD) & $\mathrm{NC}$ & $39 \%(38)$ \\
\hline \multicolumn{2}{|l|}{ Median (IQR) } & $31 \%(0-81)$ \\
\hline Total $\%$ time $\mathrm{pH}<4[\mathrm{~m}$ & SD)] & $11.4 \pm 5.9$ \\
\hline Median (IQR) & & $10.1(7.7-15.5)$ \\
\hline Esophagitis $(\%)$ grade $\mathrm{A} / \mathrm{l}$ & & $66.7 / 25.0 / 8.3$ \\
\hline Hiatal hernia $(\%)$ None $/<$ & $\geq 2 \mathrm{~cm}$ & $87.5 / 4.2 / 8.3$ \\
\hline
\end{tabular}

$S D$ standard deviation, $I Q R$ interquartile range, $N C$ diary data on PPI at baseline was not collected

10-14 days in order to reduce the chances of seroma formation. A check of the implant site is recommended 10-14 days after the procedure.

LES electrical stimulation is initiated $12 \mathrm{~h}$ after the implant procedure. The LES Stimulation System delivers therapy personalized to the individual patient's needs. Electrical stimulation is delivered using a $215-\mu$ s pulse at $20 \mathrm{~Hz}$ and $3-8 \mathrm{~mA}$ in $30 \mathrm{~min}$ sessions. The number and timing of stimulation sessions were tailored to each patient's reflux profile which was developed using 24-h 
esophageal acid exposure data and patient-reported meal and symptom times. Using the external programmer, the stimulation parameters can be adjusted, additional sessions can be added, or timing of existing sessions can be modified at patient follow-up to address any residual symptoms from initially programmed therapy. The device uses an accelerometer to detect the supine position and can deliver additional sessions to address supine/nocturnal reflux.

The battery life of the IPG with typical use is $>10$ years, after which it can be replaced using a simple outpatient procedure under local anesthesia without the need for repeat laparoscopy.

\section{Conduct of the study}

Patients who signed an informed consent form and met all the inclusion and exclusion criteria underwent laparoscopic implant of the LES stimulator as described above. The electrical stimulation therapy was initiated on day 1 post implantation and PPI therapy was discontinued. The follow-up schedule is shown in Figure 1. Adjustments of stimulation parameters were performed as needed based on any residual patient symptoms reported on follow-up and any significant acid exposure events recorded during the 24-h pH measurements.

\section{Study objectives}

The incidence of serious device- and procedure-related adverse effects was the primary safety end point, and the incidence of nonserious device- and procedure-related adverse effects was the secondary safety end point. Reduction in the patient's heartburn symptoms (calculated using the first 9 questions from the GERD-HRQL [healthrelated quality of life] questionnaire) after 6 months on LES electrical stimulation therapy compared with their baseline scores was the efficacy end point. Other efficacy end points were improvement in esophageal $\mathrm{pH}$ exposure, defined as the percentage of 24 -h that distal esophageal $\mathrm{pH}$ was $<4.0$ at 6 months post implant compared with baseline. Improvement in esophagitis, improvement in mean and end-expiratory LES pressure, improvement in daily symptom diary results, reduction in PPI usage, and improvement in SF-12 physical and mental health scores at 6 months compared to baseline for all variables were also considered as secondary efficacy end points. The $\mathrm{pH}$ data underwent a blinded review by an independent reviewer (MDC) blinded to the patient and to the time data on each $\mathrm{pH}$ study.

\section{Analysis}

22 patients were required to have a $90 \%$ chance of detecting, as significant at the $5 \%$ level, a 10-point improvement in the
GERD-HRQL score from baseline to 6 months, assuming a standard deviation of 10 points. Allowing for an approximately $10 \%$ patient dropout rate, a sample size of 25 patients was chosen as the final enrollment target for the trial.

Safety evaluation was descriptive and included the incidence, severity, and type of adverse events and clinically significant changes or abnormalities in the patient's physical examination, vital signs, clinical tests, and EKG results. Any reasons for discontinuation of study treatment were reported.

The effect of electrical stimulation of the LES on patient symptoms was measured using GERD-HRQL (on PPI and 2 weeks off PPI), symptoms and medication use reported in a daily diary, and general quality of life measured using SF-12 (on PPI and 2 weeks off PPI) using related-samples Wilcoxon signed-rank test. Change in esophagitis grade, $\%$ time distal esophageal $\mathrm{pH}$ was $<4.0$, and LES pressure and function were assessed by comparing the results at baseline to the 3-month follow-up. LES electrical stimulation therapy (EST) was evaluated using related-samples Wilcoxon signed-rank test. A $p$ value of $<0.05$ was considered statistically significant.

\section{Results}

\section{Patient characteristics}

25 GERD patients on chronic acid-suppressive therapy who met inclusion and exclusion criteria underwent successful laparoscopic implantation of the LES Stimulation System. One patient withdrew consent after 2 weeks of therapy, despite having favorable symptom response to therapy and without any side effects because of anxiety related to the device and the multiple invasive tests required by the protocol. The patient underwent an uneventful IPG explant under local anesthesia 6 weeks after implantation; the leads were left in situ. The remaining 24 patients ( 14 men, mean age $=52$ years, $\mathrm{SD}=11.6)$ were on LES EST and 23 completed their 6-month follow-up. One patient did not attend his 6-month follow-up visit. Baseline patient and disease characteristics are provided in Table 2.

\section{Safety}

A total of 32 adverse events were reported in 14 patients. One adverse event was reported as "serious adverse event not related to the device or treatment." This event was an episode of chest discomfort with mild sinus tachycardia that was not temporally related to a session of electrical stimulation. The patient reported similar uninvestigated episodes in the past, prior to starting LES electrical 
stimulation. The episode subsided spontaneously. The patient was hospitalized and an emergent cardiac catheterization was performed that revealed normal coronary anatomy. Chest X-ray revealed stable lead position in the abdominal esophagus without any evidence of migration. The patient was diagnosed with esophageal spasm, treated medically with antispasmodics, and has not had a recurrence of this symptom despite continued electrical stimulation therapy. The remaining 31 adverse events were not serious. Five events in four patients were reported as possibly or definitely device-related (three implant site pain, one localized infection, one dyspepsia) and seven events in six patients were reported as laparoscopic implant procedure-related (three implant site pain, three postoperative nausea, one localized infection). None of the patients complained of gastrointestinal side effects of bloating, inability to belch, or new dysphagia associated with LES EST throughout their 6-month follow-up.

Patients GERD symptoms as measured by GERDHRQL and daily symptom diaries

Patients GERD symptoms improved soon after initiating the electrical stimulation and continued to improve over the first 2-3 months with optimization of EST to an individual patient's needs. Patients reported better symptom control at 3 and 6 months compared to baseline on and off PPI.

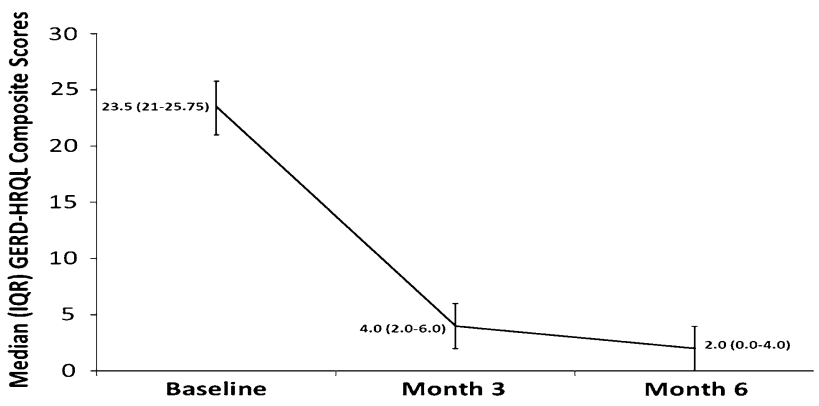

Fig. 4 Significant improvement in median (IQR) GERD-HRQL composite score at months 3 and 6 compared to baseline on-PPI and off-PPI median GERD-HRQL composite scores. There was small but statistically nonsignificant improvement from month 3 to 6

GERD-HRQL composite and individual question scores at 6 months were statistically significantly better than on- and off-PPI scores at baseline (Table 3; Fig. 4). At baseline, $92 \%$ of the subjects reported that they were "unsatisfied" with their condition off PPI and $71 \%$ on PPI compared to $4 \%$ were "unsatisfied" at their 6-month evaluation on LES EST. The patients' satisfaction with their condition at 6 months was statistically significantly better than both onPPI $(p<0.001)$ and off-PPI $(p<0.001)$ baseline satisfaction.

21 patients were available for pairwise analysis of symptoms of heartburn and regurgitation evaluated using the 14-day symptom diary. The diary data on daily patient

Table 3 Baseline and post-LES electrical stimulation therapy results

\begin{tabular}{|c|c|c|c|}
\hline & Baseline & Month 6 & $p$ value \\
\hline \multicolumn{4}{|l|}{ GERD-HRQL } \\
\hline On PPI $(n=22)$ [median (IQR)] & $9.0(6-10)$ & $2.0(0-4.0)$ & $<0.001$ \\
\hline Off PPI $(n=24)$ & $23.5(21-25.75)$ & & $<0.001$ \\
\hline \multicolumn{4}{|l|}{ SF-12 Mental Health [median (IQR)] } \\
\hline On PPI $(n=22)$ & $45.0(41.5-55.0)$ & $53.0(48.0-62.0)$ & 0.03 \\
\hline Off PPI $(n=24)$ & $49.0(35.5-54.8)$ & & 0.006 \\
\hline \multicolumn{4}{|l|}{ SF-12 Physical Health [median (IQR)] } \\
\hline On PPI $(n=22)$ & $47.0(41.8-52.8)$ & $54.0(44.0-55.8)$ & 0.11 \\
\hline Off PPI $(n=24)$ & $45.5(39.8-48.0)$ & & 0.005 \\
\hline$\%$ 24-h esophageal $\mathrm{pH}<4.0$ [median (IQR)] & $10.1(7.7-15.5)$ & $5.1(2.8-8.4)$ & $<0.001$ \\
\hline$\%$ 24-h esophageal $\mathrm{pH}<4.0$ upright & $10.4(8.0-15.2)$ & $3.2(2.5-7.4)$ & $<0.001$ \\
\hline$\%$ 24-h esophageal $\mathrm{pH}<4.0$ supine & $6.0(1.8-15.5)$ & $5.7(0.9-9.9)$ & 0.38 \\
\hline DeMeester score & $36.7(29.4-61.4)$ & $24.5(13.4-37.9)$ & 0.002 \\
\hline \multicolumn{4}{|l|}{$\%$ patients reporting heartburn affecting sleep ${ }^{a}$} \\
\hline On PPI & 70.8 & 4.3 & 0.001 \\
\hline Off PPI & 95.8 & & $<0.001$ \\
\hline \multicolumn{4}{|l|}{$\%$ patients reporting dysphagia/odynophagia ${ }^{a}$} \\
\hline On PPI & $37.5 / 25$ & $4.3 / 0$ & $0.02 / 0.13$ \\
\hline Off PPI & $66.7 / 83.3$ & & $<0.001 /<0.001$ \\
\hline
\end{tabular}

Trial design was an in-person visit (phone visit)

a Bothersome symptoms with GERD-HRQL scores $\geq 2$ on individual question 
symptoms of heartburn and regurgitation is provided in Fig. 6. There was statistically significant improvement in frequency and severity of symptoms of both heartburn and regurgitation over time with LES stimulation therapy. At baseline all patients were on antisecretory medications (proton pump inhibitors) for their GERD. At the evaluation after 6 months on LES stimulation therapy, 21 patients were able to completely stop PPI use, whereas 2 patients reported intermittent use of antisecretory medications in the 14 diary days prior to their 6-month follow-up visit. One patient each reported using PPI $>50 \%$ and $\leq 50 \%$ of the diary days.

Esophageal $\mathrm{pH}$ and high-resolution manometry

23 patients underwent esophageal $\mathrm{pH}$ testing at baseline and at 12 and 24 weeks after implant. One patient did not tolerate the tube $\mathrm{pH}$ testing at the 12-week follow-up, removed the $\mathrm{pH}$ tube a few hours after insertion, and refused repeat testing at both 12 and 24 weeks. Blinded $\mathrm{pH}$ analysis revealed that median percent time of $\mathrm{pH}<4$ in 24-h improved from $10.1 \%$ at baseline to $6.6 \%$ at 3 months to $5.1 \%$ at 6 -months $(p<0.01)$. Detailed blinded $\mathrm{pH}$ data at baseline and at 6 months are provided in Table 3 and Fig. 5.

High-resolution manometry showed no effect of LES electrical stimulation on esophageal body function and on LES residual pressure. Baseline median LES end expiratory pressure (EEP) was $10.3 \mathrm{mmHg}$ compared to the 3 -month value of $15.7 \mathrm{mmHg}(p=0.001)$. Patients' baseline median LES mean pressure (MP) was $17.8 \mathrm{mmHg}$, and at the 3 -month evaluation on LES stimulation therapy it was $22.6 \mathrm{mmHg}(p=0.002)$.

General quality of life as measured by SF-12

There was a significant improvement in the patients' general quality-of-life score as measured by SF-12 after

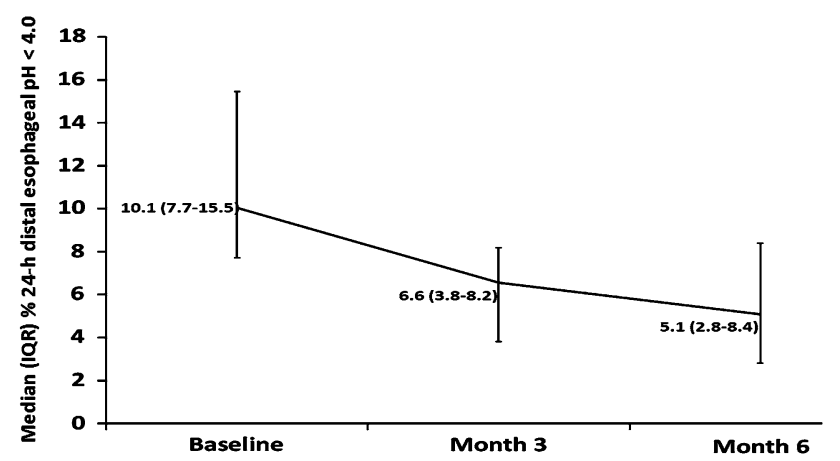

Fig. 5 Change in median IQR distal esophageal $\mathrm{pH}$ on LES electrical stimulation from baseline to 3 months $(n=24)$ and 6 months $(n=23)$. Related sample Wilcoxon signed-rank test was used to compare the $\mathrm{pH}$ at various time points
6 months on LES EST compared to their baseline scores (Table 3).

Healing of erosive esophagitis

At baseline endoscopy, 67, 25, and $8 \%$ of patients had LA grade $\mathrm{A}, \mathrm{B}$, and $\mathrm{C}$ esophagitis, respectively. At their 3 -month evaluation endoscopy, $44 \%$ of patients had no esophagitis while 35,17 , and $4 \%$ of patients had LA grade $\mathrm{A}, \mathrm{B}$, and $\mathrm{C}$ esophagitis, respectively $(p<0.01$; related sample Wilcoxon signed-rank test). Improvement in esophagitis by at least 1 grade was reported in $56 \%$ of patients at their 3-month endoscopy.

\section{Discussion}

This is the first human trial of long-term electrical stimulation as a treatment for GERD. The results show that electrical stimulation of the LES can safely improve symptoms of GERD as measured by GERD-HRQL and the patient symptoms diary, reduce esophageal acid exposure by augmenting esophageal sphincter pressures, and eliminate the need for PPI medications without the GI side effects typically seen with other antireflux procedures that involve mechanical alteration of the gastroesophageal junction. LES EST was not associated with any adverse effects or sensation and specifically did not interfere with physiological LES relaxation and hence there was no dysphagia.

GERD is "a condition which develops when the reflux of stomach contents causes troublesome symptoms and/or complications" [9]. Society of American gastrointestinal endoscopic surgeons guidelines for surgical treatment of GERD recommend surgery for individuals who have failed medical management (inadequate symptom control, severe regurgitation not controlled with acid suppression, or medication side effects) or opt for surgery despite successful medical management (due to quality-of-life considerations, lifelong need for medication intake, expense of medications) [3]. On the other hand, the AGA position statement on management of GERD recommends antireflux surgery for GERD patients who are responsive to but intolerant of acid suppressive therapy or for patients with persistent and troublesome GERD symptoms despite medical therapy. The AGA statement warns of deleterious GI side effects from an antireflux procedure [10].

Limitations and concerns with current medical and surgical treatments leave a significant GERD patient population whose symptoms are not adequately controlled with medical management and seek a valid therapeutic option as an alternative to surgical fundoplication [11-13]. This has led to a search for an alternative, less invasive treatment for 

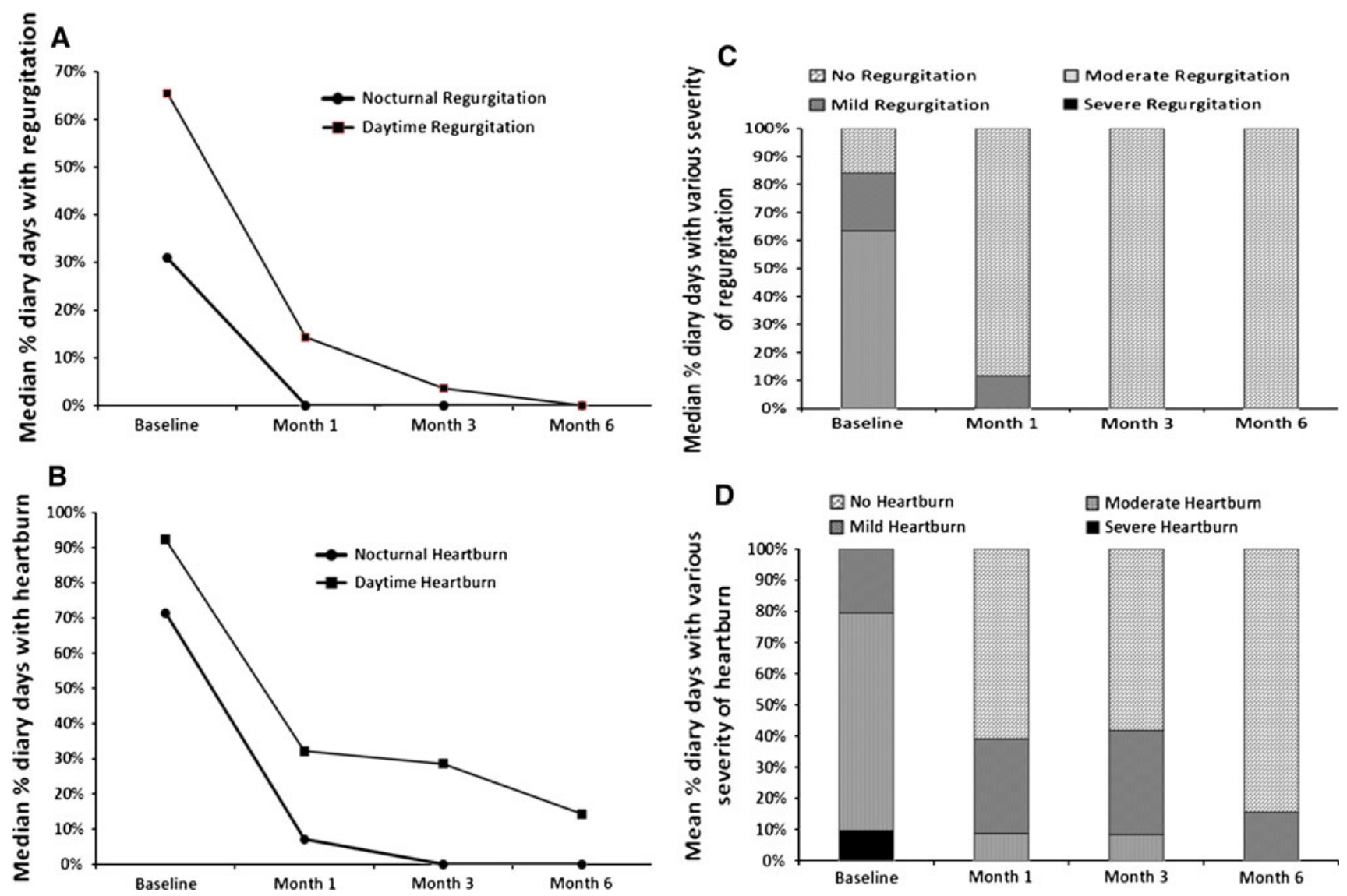

Fig. 6 Median \% daily diary days with symptoms of nocturnal and daytime regurgitation (A) and heartburn (B), and \% of the diary days with various severity levels of regurgitation $(\mathbf{C})$ and heartburn $(\mathbf{D})$ at 1, 3, and 6 months compared to the baseline

GERD that would address all the symptoms of GERD without deleterious side effects. Various endoscopic methods have been developed and approved but only a few are currently available for clinical use [14]. Most of these therapies were removed from the market due to lack of efficacy or serious adverse events. Currently approved as an alternative therapy for GERD is Esophy $X^{\circledR}$ TIF $^{\circledR}$ (transoral incisionless fundoplication) (EndoGastric Solutions, Redmond City, CA), which uses T-tags in an attempt to create an endoscopic fundoplication, and the LINX reflux management system (Torax Medical, Inc., Shoreview, MN), which uses a bracelet of magnets encased in titanium placed around the LES to create a "magnetic sphincter" $[15,16]$.

Treatment goals established by the SAGES for an antireflux device include elimination of symptoms, healing of esophagitis, prevention of complications, and maintenance of remission [3]. Our results suggest that application of long-term electrical stimulation to the LES using the permanently implanted LES Stimulation System may fulfill all these goals. LES stimulation resulted in significant enhancement of LES pressure and significant reduction in distal esophageal acid exposure, which in turn resulted in a significant improvement in GERD symptoms and patient quality of life. The improvement in symptoms was sustained over the 6-month follow-up period and continued to improve over time due to optimization of therapy to address each patient's symptoms without any signs of adaptation and/or muscle fatigue. Our patients reported better GERD-HRQL scores and better satisfaction with LES EST than baseline on-PPI scores as LES electrical stimulation addressed global GERD symptoms, including regurgitation. Regurgitation is a symptom usually not responsive to medical therapy and has been cited as an appropriate indication for an antireflux procedure. There was a complete elimination of the symptom of regurgitation at the 6-month evaluation on LES stimulation as reported in the daily diaries. There was significant healing of erosive esophagitis at the 3-month endoscopy.

Most importantly, electrical stimulation of the LES had no effect on LES residual pressure, and none of the patients in this trial reported dysphagia or any other GI symptoms on LES stimulation therapy. GI side effects such as dysphagia, gas bloat, flatulence, and diarrhea have been the Achilles heel of all antireflux procedures. Significant postoperative rates of dysphagia with antireflux surgery, 
requiring endoscopic dilation, ranging from 0 to $25 \%$ and a reoperation rate ranging from 1.8 to $10.8 \%$ have been reported [3]. Significantly higher levels of bloating and flatulence are also reported with antireflux surgery compared to antisecretory therapy over long-term (5-year) follow-up [18]. Of particular importance is the excellent safety profile of LES electrical stimulation therapy. There have been no device- or procedure-related serious adverse effects during implantation or during the 6-month study. There were a few minor anticipated adverse effects reported with the surgical implant procedure, which is expected. Previous experience with electrical stimulation of the stomach, using comparable electrodes and devices for the purpose of treating gastroparesis, diabetes, and obesity, has also shown an excellent safety and side-effect profile [19]. This improved safety profile of LES electrical stimulation may result in better patient acceptance of EST as an antireflux procedure and expand this option to a larger group of patients suffering from refractory GERD.

Another issue with antireflux surgery is the learning curve. Antireflux surgery performed by less experienced individuals can result in suboptimal failure rates, complications, reoperations, operative time, hospital days, and conversion to open surgery [20, 21]. Reports suggest that outcomes of high-volume referral centers are significantly better than those seen in low-volume centers $[13,18]$. The LES stimulation implant procedure is quiet simple and can easily be standardized for uniform performance by general laparoscopic surgeons. The ability to optimize the therapy noninvasively over time allows for fine-tuning of GERD therapy at patient follow-up to continually improve GERD outcomes, an option not available with current endoscopic or laparoscopic antireflux procedures.

There are limitations to this study. We enrolled patients who were at least partial responders to PPIs and our results may not apply to patients who are complete nonresponders to PPI therapy. However, symptoms of nonresponders to PPI maybe caused by nonreflux conditions such as visceral hypersensitivity or an esophageal motility disorder and they may not respond to antireflux therapy. Because of these reasons, response to medical therapy has been commonly used to select patients for both endoscopic and surgical antireflux procedures. Also, this was an open-label study and hence the placebo effect may have contributed to improvement in symptoms and a "regression to mean" effect could have contributed to improvement in physiological variables such as esophageal $\mathrm{pH}$.

In addition, patients with severe LES dysfunction (LES end-expiratory pressure $<5 \mathrm{mmHg}$ ), significant hiatal hernia of $>3 \mathrm{~cm}$, and grade D esophagitis were not evaluated in this trial. These patients usually have severe LES dysfunction or a significant alteration in their gastroesophageal anatomy that may not be amenable to correction with electrical stimulation of the LES. Patients who have moderate anatomical disruption of the GEJ anatomy, i.e., hiatal hernia of 3-5 cm, could be addressed with a combination of restoration of abdominal esophagus, repair of the diaphragmatic hiatus, and implant of the LES stimulator, thus avoiding fundoplication and reducing long-term side effects associated with the fundoplication. However, this technique needs to be evaluated in a prospective clinical trial.

Future improvements to the surgical implant procedure, including options for single-port, periumbilical single-site access and use of mainly 3-mm trocars and instruments, may further improve patient acceptability of the surgical procedure. In leaner patients with a normal, nonfatty liver, the liver retractor can be replaced with a V-shaped extracorporeal knotted thread. Moreover, by using a longer lead body, the skin incision for the subcutaneous stimulator could be made below the "bikini line" for better cosmetic outcome.

In conclusion, our long-term results show that electrical stimulation of the LES using the laparoscopically implanted LES Stimulation System is safe and effective in the treatment of GERD. LES EST results in significant improvement in patient GERD symptoms of heartburn and regurgitation, reduction in GERD medication use, reduction in esophageal acid exposure, and improvement in LES pressures without causing adverse sensation or symptoms. The improvement in patient outcome is sustained over time. Furthermore, LES EST can be optimized noninvasively to an individual patient's disease profile and changing needs over time to achieve sustained improvement in patient outcomes.

Disclosures Research support for this study was provided by EndoStim BV, Hague, the Netherlands. Dr. Michael D. Crowell is a consultant for EndoStim Inc. Leonardo Rodríguez, Patricia Rodriguez, Beatriz Gómez, Oksenberg Danny, Juan C. Ayala, Alberto Perez-Castilla, and Manoel G.Netto have no conflicts of interest or financial ties to disclose.

\section{References}

1. Sandler RS, Everhart JE, Donowitz M et al (2002) The burden of selected digestive diseases in the United States. Gastroenterology 122:1500-1511

2. Everhart JE (ed) (1994) Digestive diseases in the United States: epidemiology and impact. US Department of Health and Human Services, Public Health Service, National Institutes of Health, National Institute of Diabetes and Digestive and Kidney Diseases. Washington, DC: US Government Printing Office, NIH publication No. 94-1447

3. Stefanidis D, Hope WW, Kohn GP et al (2010) Guidelines for surgical treatment of gastroesophageal reflux disease. Surg Endosc 24:2647-2669

4. American Gastroenterological Association (2008) GERD patient study: patients and their medications. Harris Interactive Inc., New York 
5. Ellis F, Berne TV, Settevig K (1968) The prevention of experimentally induced reflux by electrical stimulation of the distal esophagus. Am J Surg 115:482-487

6. Clarke JO, Jagannath SB, Kalloo AN et al (2007) An endoscopically implantable device stimulates the lower esophageal sphincter on demand by remote control: a study using a canine model. Endoscopy 39:72-76

7. Sanmiguel CP, Hagiike M, Mintchev MP et al (2008) Effect of electrical stimulation of the LES on LES pressure in a canine model. Am J Physiol Gastrointest Liver Physiol 295:389-394

8. Rodríguez L, Rodríguez P, Neto MG et al (2012) Short-term electrical stimulation of the lower esophageal sphincter increases sphincter pressure in patients with gastroesophageal reflux disease. Neurogastroenterol Motil 24:446-450

9. Vakil N, van Zanten SV, Kahrilas P et al (2006) The montreal definition and classification of gastroesophageal reflux disease: a global evidence-based consensus. Am J Gastroenterol 101:1900-1920

10. Kahrilas PJ, Shaheen NJ, Vaezi MF et al. (2008) American Gastroenterological Association medical position statement on the management of gastroesophageal reflux disease. Gastroenterology 135:1383-1391, 1391.e1-5

11. Lodato F, Azzaroli F, Turco L et al (2010) Adverse effects of proton pump inhibitors. Best Pract Res Clin Gastroenterol 24(2): 193-201

12. Spechler SJ, Lee E, Ahnen D et al (2001) Long-term outcome of medical and surgical therapies for gastroesophageal reflux disease. Follow-up of a randomized controlled trial. JAMA 285: 2331-2338

13. Vakil N, Shaw M, Kirby R (2003) Clinical effectiveness of laparoscopic fundoplication in a US community. Am J Med 114:1-5
14. Rothstein RI (2008) Endoscopic therapy of gastroesophageal reflux disease: outcomes of the randomized-controlled trials done to date. J Clin Gastroenterol 42:594-602

15. Cadière GB, Van Sante N, Graves JE et al (2009) Two-year results of a feasibility study on antireflux transoral incisionless fundoplication using EsophyX. Surg Endosc 23:957-964

16. Bonavina L, DeMeester T, Fockens P et al (2010) Laparoscopic sphincter augmentation device eliminates reflux symptoms and normalizes esophageal acid exposure: one- and 2-year results of a feasibility trial. Ann Surg 252:857-862

17. Banerjee R, Pratap N, Kalapala R, Reddy DN (2010) In patients with GERD, electrical stimulation therapy (EST) significantly and consistently increases lower esophageal sphincter (LES) pressure. J Gastroenterol Hepatol 25:A16

18. Galmiche JP, Hatlebakk J, Attwood S et al (2011) laparoscopic antireflux surgery versus esomeprazole treatment for chronic GERD. the LOTUS randomized clinical trial. JAMA 305:1969-1977

19. McCallum RW, Zhiyue L, Forester J et al (2011) Gastric electrical stimulation improves outcomes of patients with gastroparesis for up to 10 years. Clin Gastroenterol Hepatol 9:314-319

20. Watson DI, Baigrie RJ, Jamieson GG (1996) A learning curve for laparoscopic fundoplication. Definable, avoidable, or a waste of time? Ann Surg 224:198-203

21. Deschamps C, Allen MS, Trastek VF, Johnson JO, Pairolero PC (1998) Early experience and learning curve associated with laparoscopic Nissen fundoplication. J Thorac Cardiovasc Surg 115:281-284 discussion 284-285 